\title{
Resultados a longo prazo do uso de drogas antiproliferativas na trabeculectomia primária
}

\author{
Long-term outcomesof antiproliferativeagents in primary trabeculectomy
}

\author{
Eleonore J. Norris ${ }^{1}$ \\ Joyce C. Schiffman ${ }^{2}$ \\ Paul F. Palmberg ${ }^{3}$ \\ Paulo Augusto de Arruda Mello ${ }^{4}$
}

\section{RESUMO}

Objetivo: Avaliar comparativamente a longo prazo os resultados cirúrgicos de trabeculectomias nas quais foram utilizadas injeções subconjuntivais de 5-fluorouracil (5-FU) no pós-operatório ou aplicações de mitomicina $\mathrm{C}(\mathrm{MMC})$ peroperatória. Métodos: Estudo retrospectivo de 133 olhos submetidos a trabeculectomia primária. Resultados: A pressão ocular não apresentou valores estatisticamente significantes em nenhuma das visitas de pós-operatório quando comparados os grupos em que se utilizou a mitomicina $\mathrm{C}$ ou 5-fluorouracil. Em cada um dos grupos analisados separadamente, os níveis da pressão ocular não apresentaram diferenças estatisticamente significantes nos períodos pré e pós-operatório. A incidência de complicações pós-operatórias também não apresentou diferença estatisticamente significante à exceção de alterações epiteliais na córnea, mais freqüentes no grupo tratado com 5-fluorouracil. Nos dois grupos, destacou-se a elevada incidência de desenvolvimento/progressão de catarata. Conclusão: $\mathrm{O}$ uso de mitomicina $\mathrm{C}$ ou 5-fluorouracil promoveu redução importante e estável da pressão ocular, com índices de complicações semelhantes.

Descritores: Trabeculectomia; Glaucoma/cirurgia; Pressão intra-ocular/efeitos de drogas Campos visuais; Fluorouracil/administração \& dosagem; Mitomicinas/administração \& dosagem; Mitomicinas/uso terapêutico; Fluorouracil/uso terapêutico; Estudos retrospectivos

\section{INTRODUÇÃO}

A principal causa de insucesso da cirurgia fistulante é a cicatrização por proliferação de tecido conjuntivo no sítio de filtração. O uso de agentes antiproliferativos promove uma melhora nos índices de sucesso desta cirurgia por inibir a proliferação fibroblástica e a biosíntese de colágeno e material extracelular ${ }^{(1-2)}$.

O 5-Fluorouracil (5-FU) é uma droga capaz de inibir a proliferação de fibroblastos in vitro e in vivo, reduzindo a síntese de colágeno e a formação da cicatriz ${ }^{(3-4)}$. A droga pode ser injetada no espaço subconjuntival no período pós-operatório ${ }^{(5)}$, ou aplicada sob o retalho conjuntival durante o procedimento cirúrgico. Apesar dos efeitos benéficos dessa droga já terem sido demonstrados nos vários tipos de glaucoma ${ }^{(6)}$, diversos problemas estão associados à sua administração, como a necessidade de injeções freqüentes, toxicidade epitelial à córnea, vazamento na incisão conjuntival e endoftalmite ${ }^{(7-8)}$.

A mitomicina $\mathrm{C}(\mathrm{MMC})$ é um agente antiproliferativo aplicado em dose única per-operatória. Tem sido usada como alternativa ao 5-FU por não ser necessário as múltiplas injeções subconjuntivais. A sua efetividade foi demonstrada em inúmeros estudos clínicos e experimentais ${ }^{(1,7,9)}$. Entretanto 
há referência de maior incidência de complicações como hipotensão ocular e endoftalmite ${ }^{(10-11)}$.

$\mathrm{O}$ uso de 5-FU ou MMC na cirurgia para glaucoma se popularizou na década de 90 . É de conhecimento geral que tais drogas promovem um melhor controle da pressão intra-ocular (Po) a médio prazo com uma menor porcentagem de pacientes necessitando de medicação antiglaucomatosa suplemen$\operatorname{tar}^{(5,12)}$. O propósito do presente estudo é analisar resultados a longo prazo de trabeculectomias primárias com 5-FU ou MMC e determinar os benefícios em termos de preservação do campo visual, assim como as complicações advindas do uso destes agentes.

\section{MÉTODOS}

Este estudo foi aprovado pela comissão de pesquisa da Universidade de Miami e pela comissão de ética médica do Hospital São Paulo/UNIFESP-EPM.

Foram analisados prontuários de 986 pacientes submetidos a trabeculectomia no Bascom Palmer Eye Institute, Departamento de Oftalmologia da Universidade de Miami, Flórida entre janeiro/1989 e dezembro/1995. A indicação de cirurgia ocorreu em casos onde a Po atingiu níveis inaceitáveis apesar do uso de medicação máxima tolerada, considerando as alterações de disco óptico e defeitos campimétricos.

Foi incluída na análise a primeira cirurgia incisional para controle do glaucoma (trabeculectomia primária) realizada por um dos autores (PFP), utilizando técnica de incisão valvular descrita previamente ${ }^{(13)}$. Critérios de exclusão foram: 1) Portadores de glaucoma secundário, exceto os glaucomas cortisônico, pigmentar e pseudoexfoliativo, 2) Portadores de malformações oculares congênitas, 3) Cirurgia conjuntival e/ou intra-ocular incisional prévia, 4) Portadores de doença ocular que simulasse defeito glaucomatoso em campo visual, 5) Acompanhamento pós-operatório menor que 12 meses, 6) Cirurgias combinadas de catarata e glaucoma. Em casos onde a cirurgia foi realizada em ambos os olhos, foi incluído o olho com maior tempo de seguimento.

Das cirurgias realizadas entre janeiro/1989 e abril/1991 foram selecionados prontuários de 38 pacientes submetidos a trabeculectomia primária com injeções pós-operatórias suplementares de $5-\mathrm{FU}(5 \mathrm{mg} / 0,1 \mathrm{ml})$ a $180^{\circ}$ da bolha filtrante, num total de cinco aplicações. A primeira injeção foi dada imediatamente após a cirurgia e quatro outras injeções foram aplicadas durante os primeiros 14 dias de pós-operatório. A droga só foi interrompida precocemente com o surgimento de alterações severas da córnea, como ceratopatia punteada ou extensas desepitelizações.

Das cirurgias subseqüentes, foram selecionados 95 pacientes submetidos a trabeculectomia primária com aplicação per-operatória de MMC. Neste grupo foi aplicada uma esponja tipo "weck-cell” embebida em solução de $0,5 \mathrm{mg} / \mathrm{ml}$ de MMC por 5 minutos sobre a esclera, com irrigação copiosa subseqüente de solução salina balanceada (BSS).
Após a cirurgia, nos dois grupos, foi utilizado colírio de corticosteróide que foi reduzido gradualmente de acordo com a reação inflamatória. Lise de sutura da incisão escleral no pós-operatório foi realizada quando a Po atingia valores acima de $21 \mathrm{mmHg}$ ou quando a bolha filtrante não era evidente.

Foram obtidos do prontuário dados como idade, sexo, raça, tipo de glaucoma, droga antiproliferativa empregada e informações pré e pós-operatórias de acuidade visual (AV), valores da Po, MD (mean deviation) e PSD (pattern standard deviation) dos exames de perimetria computadorizada, e uso de medicações antiglaucomatosas. Foram também registradas as complicações per e pós-operatórias.

Os resultados foram expressos como média \pm desvio padrão. A análise estatística incluiu o teste " $t$ " de Student, o quiquadrado $\left(\chi^{2}\right)$, o Mann-Whitney e o teste "log rank". O teste de sobrevivência de Kaplan-Meier foi empregado para avaliar a probabilidade de sucesso do controle da Po ao longo do tempo. Para esta análise, o sucesso cirúrgico foi classificado em duas categorias: Po $\leq 15 \mathrm{mmHg}$ e Po $\leq 21 \mathrm{mmHg}$, independentemente do uso de medicação suplementar.

\section{RESULTADOS}

Os dados pré-operatórios dos dois grupos estudados encontram-se resumidos na tabela 1 . Não foi observada diferença estatisticamente significante em relação à idade, sexo, raça, Po, tratamento clínico/laserterapia, acuidade visual (AV) e parâmetros de campo visual (MD e PSD) entre eles.

A Po pré e pós-operatória dos grupos 5-FU e MMC pode ser observada na tabela 2. A redução da Po no pós-operatório

\begin{tabular}{|c|c|c|c|}
\hline \multicolumn{4}{|c|}{ Tabela 1. Dados pré-operatórios } \\
\hline & $5-F U$ & MMC & \multirow[t]{2}{*}{$\mathbf{p}$} \\
\hline & $\mathbf{N} \%$ & $\mathbf{N} \%$ & \\
\hline \multicolumn{4}{|l|}{ Idade (anos) } \\
\hline Média \pm desvio padrão & $67 \pm 12$ & $64 \pm 15$ & $0,28^{*}$ \\
\hline \multicolumn{4}{|l|}{ Raça } \\
\hline Brancos & $34(89,5)$ & $73(76,8)$ & $0,16^{\star *}$ \\
\hline Negros & $04(10,5)$ & $22(23,2)$ & \\
\hline \multicolumn{4}{|l|}{ Sexo } \\
\hline Masculino & $25(65,8)$ & $58(61,1)$ & $0,76^{* *}$ \\
\hline Feminino & $13(34,2)$ & $37(38,9)$ & \\
\hline \multicolumn{4}{|l|}{ Tipo de glaucoma } \\
\hline Primário de ângulo aberto & $24(63,2)$ & $70(73,7)$ & \\
\hline Crônico de ângulo estreito & $04(10,5)$ & $09 \quad(9,5)$ & \\
\hline Pseudoexfoliativo & $08(21,1)$ & $08 \quad(8,4)$ & $0,20^{* *}$ \\
\hline Pigmentar & $02(5,3)$ & $06 \quad(6,3)$ & \\
\hline Outros & - & $02 \quad(2,1)$ & \\
\hline \multicolumn{4}{|l|}{ Tratamento prévio } \\
\hline $\mathrm{N}^{\circ}$ medicações (média \pm d.p) & $2,71 \pm 1,0$ & $2,41 \pm 1,1$ & $0,25^{\star * \star}$ \\
\hline Iridotomia & $4(10,5)$ & $5 \quad(6,3)$ & $0,41^{* * *}$ \\
\hline Trabeculoplastia & $29(76,3)$ & $54(56,8)$ & $0,36^{\star * *}$ \\
\hline Seguimento (meses) & $64 \pm 32$ & $48 \pm 17$ & $<0,001^{\#}$ \\
\hline
\end{tabular}




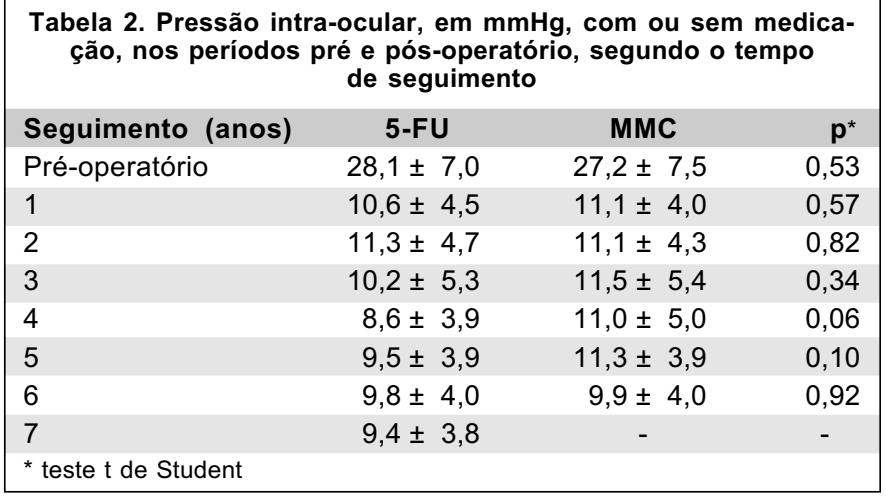

foi estatisticamente significante nos dois grupos, não havendo diferença estatisticamente significante da Po pós-operatória em cada grupo nos diversos tempos de acompanhamento e na comparação entre eles. Para ajuste da Po no período pósoperatório, utilizou-se de procedimentos como a lise de sutura e o agulhamento. No grupo de 5-FU, as incidências foram, respectivamente, $66 \%$ ( 25 olhos) e $18 \%$ ( 7 olhos). No grupo de MMC, realizou-se lise de sutura em 59 olhos (62\%) e agulhamento em 25 olhos (26\%).

A figura 1 ilustra a curva de sobrevida de Kaplan Meier, que demonstra uma resposta bastante semelhante nas duas modalidades de tratamento. Quando foram utilizadas Po $<15 \mathrm{mmHg}$ e Po $<21 \mathrm{mmHg}$ como ponto de corte, a comparação entre as probabilidades de sucesso não se evidenciou estatisticamente significante $(p=1,00$ e $p=0,72$, respectivamente).

Analisando os valores de MD e PSD obtidos ao longo do

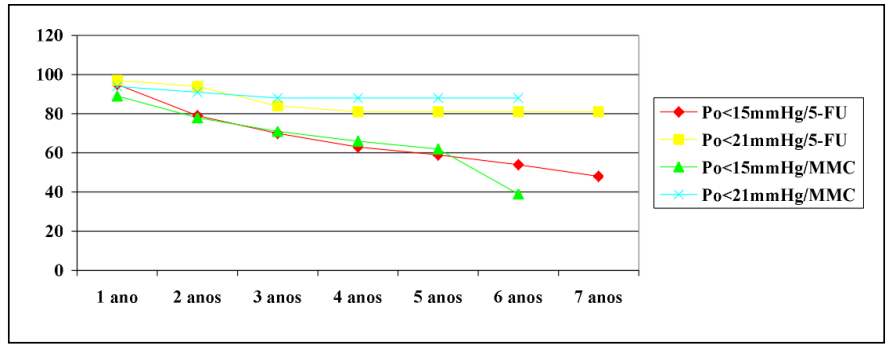

Figura 1 - Curva de sobrevida de Kaplan Meier

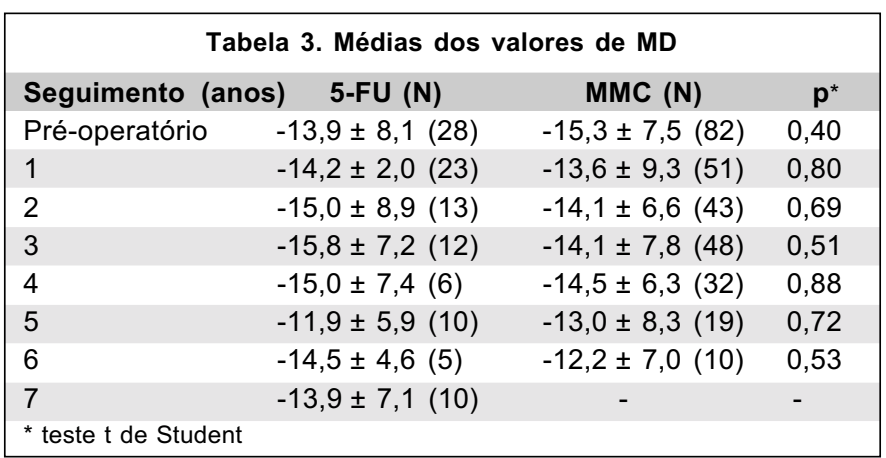

seguimento, observa-se que mantiveram a estabilidade, se comparados aos valores pré-operatórios. (Tabelas 3 e 4 )

A tabela 5 mostra a freqüência das principais complicações pós-operatórias observadas no presente estudo. Observa-se uma maior incidência de alterações epiteliais corneanas (estatisticamente significante) e descolamento de coróide no grupo tratado com 5-FU e maior incidência de maculopatia por hipotonia e desenvolvimento e/ou progressão de catarata no grupo tratado com MMC (estatisticamente não significante).

Dentre os 16 olhos que apresentaram descolamento de coróide pós-operatório, em três olhos o quadro persistiu por um período mais prolongado, sendo necessária a drenagem dos bolsões (um no grupo tratado com 5-FU e dois no tratado com MMC).

Blebite ocorreu num total de seis olhos $(4,5 \%)$, em média 36 meses após a cirurgia (de um a 86 meses). Destes, três evoluíram para endoftalmite, todos do grupo tratado com MMC. Dois deles foram submetidos à vitrectomia com injeção intravítrea de antibióticos e em um deles foram realizadas apenas aspiração vítrea e injeção intravítrea de antibióticos.

\section{DISCUSSÃO}

O objetivo da trabeculectomia é a redução da Po a níveis que venham a prevenir futuros danos ao nervo óptico e progressão de defeitos de campo visual. A proliferação fibroblástica é considerada a causa mais comum da falência de bolsas filtrantes em olhos trabeculectomizados. Por isso, o uso de agentes antiproliferativos em cirurgias para o glaucoma vem sendo investigado há algum tempo.

\begin{tabular}{|lccc|}
\hline \multicolumn{4}{|c|}{ Tabela 4. Médias dos valores de PSD } \\
Seguimento (anos) & $\mathbf{5 - F U}(\mathbf{N})$ & MMC (N) & $\mathbf{p}^{*}$ \\
\hline Pré-operatório & $7,2 \pm 3,8(28)$ & $7,8 \pm 3,3(82)$ & 0,38 \\
1 & $6,9 \pm 3,6(23)$ & $7,7 \pm 3,5(51)$ & 0,34 \\
2 & $7,3 \pm 3,0(13)$ & $8,1 \pm 3,6(43)$ & 0,45 \\
3 & $7,5 \pm 2,6(12)$ & $8,1 \pm 3,8(48)$ & 0,23 \\
4 & $5,9 \pm 2,5(6)$ & $8,1 \pm 3,1(32)$ & 0,20 \\
5 & $7,5 \pm 3,2(10)$ & $7,6 \pm 3,8(19)$ & 0,95 \\
6 & $10,5 \pm 2,8(5)$ & $7,5 \pm 4,1(10)$ & 0,18 \\
7 & $7,3 \pm 3,7(10)$ & - & - \\
${ }^{*}$ teste $t$ de Student & & & \\
\hline
\end{tabular}

\begin{tabular}{|c|c|c|c|c|c|}
\hline & \multicolumn{2}{|c|}{ 5-FU } & \multicolumn{2}{|c|}{ MMC } & \multirow[t]{2}{*}{$\mathbf{p}^{* *}$} \\
\hline & $\mathbf{N}$ & $(\%)$ & $\mathbf{N}$ & $(\%)$ & \\
\hline Defeito epitelial & 6 & 15,8 & 2 & 2,1 & $0,01^{\#}$ \\
\hline Seidel + & 7 & 18,4 & 12 & 12,6 & 0,56 \\
\hline Descolamento de coróide & 7 & 18,4 & 9 & 9,5 & 0,26 \\
\hline Catarata & 14 & 36,8 & 54 & 56,8 & 0,06 \\
\hline Maculopatia por hipotonia & 2 & 5,3 & 7 & 7,4 & 0,96 \\
\hline Blebite & 2 & 5,3 & 4 & 4,2 & 1,00 \\
\hline Endoftalmite & 0 & - & 3 & 3,1 & 0,64 \\
\hline${ }^{* *}$ teste $\chi^{2}$ & & & & & \\
\hline
\end{tabular}


Alguns autores ${ }^{(2,6)}$ demonstraram claramente que tanto o 5-FU quanto a MMC melhoram o prognóstico cirúrgico em pacientes com alto risco de falência, em tempos de acompanhamento curtos. Outros ${ }^{(1,8)}$ sugerem que pacientes submetidos a cirurgia fistulante com MMC têm, a longo prazo, maior probabilidade de controle pressórico sem medicação antiglaucomatosa auxiliar quando comparados com aqueles que utilizaram na cirurgia o 5-FU. No presente estudo foram avaliadas trabeculectomias primárias (primeira cirurgia intra-ocular), cirurgias consideradas como de baixo risco. Em concordância com a literatura, não foi observada diferença no controle da Po entre os dois grupos em nenhum dos intervalos estudados, durante os seis anos de acompanhamento. A freqüência de procedimentos auxiliares (lise de sutura e agulhamento) no controle da Po também não diferiu nos dois grupos, sugerindo uma evolução pós-operatória semelhante.

Ao se analisar a freqüência de Po inferior a $15 \mathrm{mmHg}$ nos diversos intervalos de acompanhamento, nos dois grupos, também não se detectou diferença estatística. Este valor de Po $(15 \mathrm{mmHg}$ ) foi escolhido em conseqüência ao trabalho realizado por Odberg ${ }^{(14)}$, que sugere que a Po nestes níveis é, na maioria das vezes, adequada para evitar a progressão da lesão glaucomatosa.

Os índices de complicações foram semelhantes nos dois grupos, a exceção da maior incidência de defeitos epiteliais corneanos e descolamento de coróide do grupo onde se utilizou o 5-FU. Outras complicações sérias, como endoftalmite e maculopatia por hipotonia, foram mais freqüentes no grupo onde se utilizou a MMC, mas sem significância estatística. Existe uma preocupação teórica que poderia haver um aumento na incidência de endoftalmite em trabeculectomias realizadas com agentes antiproliferativos, por estes proporcionarem bolhas fistulantes de paredes mais delgadas e com maior probabilidade de apresentarem vazamento (Seidel + ). Os achados do presente estudo podem ser comparados favoravelmente a outros previamente descritos ${ }^{(1,6-7)}$.

O surgimento/progressão de catarata foi a causa mais freqüente de redução de acuidade visual. Analisando os índices de pacientes que foram submetidos à cirurgia de catarata isoladamente ou em conjunto com aqueles que sofreram redução de ao menos três linhas de AV na tabela de Snellen, não se observou diferença significante entre os grupos 5-FU e MMC.

\section{CONCLUSÃO}

Os resultados do presente estudo mostraram que tanto o 5FU quanto a MMC proporcionaram valores baixos e estáveis da Po, num acompanhamento de seis anos, em cirurgias de baixo risco. Também pode ser observado, como na literatura ${ }^{(2,6,8)}$, que as incidências de complicações sérias, apesar de maiores no grupo $\mathrm{MMC}$, não apresentaram diferenças estatisticamente significantes quando comparadas ao grupo 5-FU. Complicações pós-operatórias em cirurgias fistulantes existem inde- pendentemente do uso de agentes antiproliferativos. Enquanto o uso de MMC parece ser efetivo em promover uma adequada bolha filtrante, ainda são necessários estudos para melhor conhecimento da concentração e/ou tempo de exposição adequados na aplicação subconjuntival, e para avaliação da eficácia e segurança deste método a longo prazo. Baseado em nossos dados de menor toxicidade à córnea, e na vantagem de administração única e per-operatória da MMC, acreditamos que este agente seja uma alternativa viável ao uso de injeções subconjuntivais de 5-FU, mas não descartamos o uso do último em casos de cirurgia de baixo risco por apresentar redução da Po e índices de complicações semelhantes.

\section{ABSTRACT}

Purpose: To evaluate and compare long-term outcomes of trabeculectomies with subconjunctival injections of 5-fluorouracil (5-FU) after sugery or use of mitomycin C (MMC) during surgery. Methods: Retrospective study of 133 eyes who underwent primary trabeculectomy. Results: Intraocular pressure levels were not statistically significant at any of the postoperative visits when comparing the 5-fluorouracil and mitomycin $\mathrm{C}$ groups. In each group, analyzed separately, the intra-ocular pressure levels showed no statistically significant difference before or after surgery. The incidence of postoperative complications was not statistically significant as well, except for a greater incidence of the corneal epithelial defects, more frequent in the 5-fluorouracil group. In both groups, a greater incidence of development/progression of cataract was noted. Conclusion: Both mitomycin $\mathrm{C}$ and 5-fluorouracil promoted an important and stable decrease in intraocular pressure with similar complication rates.

Keywords: Trabeculectomy; Glaucoma/surgery; Intraocular pressure/drug effects; Visual fields; Fluorouracil/administration \& dosage; Mitomycins/administration \& dosage; Fluorouracil/therapeutic use; Mitomycins/therapeutic use; Retrospective studies

\section{REFERÊNCIAS}

1. Kitazawa Y, Kawase K, Matsushita H, Minobe M. Trabeculectomy with mitomycin. A comparative study with fluorouracil. Arch Ophthalmol 1991;109:1693-8.

2. Susanna Jr. R, \& Takahashi WY. Estudo comparativo entre o uso de 5fluorouracil e da mitomicina em olhos trabeculectomizados. Arq Bras Oftalmol 1993;56:25-8.

3. Binder S, Riss B, Skorpik C, Kulnig W. Inhibition of experimental intraocular proliferation with intravitreal 5-fluorouracil. Graefe's Arch Clin Exp Ophthalmol 1983;221:126-9.

4. Yamamoto T, Varani J, Soong HK, Lichter PR. Effects of 5-fluorouracil and mitomycin $\mathrm{C}$ in cultured rabbit subconjuntival fibroblasts. Ophthalmology 1990;97:1204-10.

5. Goldenfeld M, Krupin T, Ruderman JM, Wong PC, Rosenberg LS, Ritch R, et al. 5-Fluorouracil in initial trabeculectomy. A prospective, randomized, multicenter study. Ophthalmology 1994;101:1024-9. 
6. Prata Jr. JA, Seah SKL, Minckler DS, Baerveldt G, Lee PP, Heuer DK. Postoperative complications and short-term outcome after 5-fluorouracil or mitomycin-C trabeculectomy. J Glaucoma 1995;4:25-31.

7. Skuta GL, Beeson CC, Higginbotham EJ, Lichter PR, Musch DC, Bergstrom $\mathrm{TJ}$, et al. Intraoperative mitomycin versus postoperative 5 -fluorouracil in high-risk glaucoma filtering surgery. Ophthalmology 1992;99:438-44.

8. Katz GJ, Higginbotham EJ, Lichter PR, Skuta GL, Musch DC, Bergstrom $\mathrm{TJ}$, et al. Mitomycin C versus 5-Fluorouracil in high-risk glaucoma filtering surgery. Extended follow-up. Ophthalmology 1995;102:1263-9.

9. Palmer SS. Mitomycin as an adjunct chemotherapy with trabeculectomy. Ophthalmology 1991;98:317-21.

10. Parrish II RK, Minckler D. "Late endophthalmitis" - filtering surgery time bomb? Ophthalmology 1996;103:1167-8.
11. Singh K, Byrd S, Egbert PR, Budenz D. Risk of hypotony after primary trabeculectomy with antifibrotic agents in a black West African population. J Glaucoma 1998;7:82-5

12. Costa VP, Comegno PEC, Vasconcelos JPC, Malta RFS, José NK. Low-dose mitomycin C trabeculectomy in patients with advanced glaucoma. J Glaucoma 1996;5:193-9.

13. Suñer IJ, Greenfield DS, Miller MP, Nicolela MT, Palmberg PF. Hypotony maculopathy after filtering surgery with mitomycin $\mathrm{C}$. Incidence and treatment [commented on Ophthalmology 1997;104:1367]. Ophthalmology 1997; 104:207-15.

14. Odberg T. Visual field prognosis in advanced glaucoma. Acta Ophthalmol 1987;65 (182 Suppl):27.

\section{ABO ELETRÔNICO}

\section{A versão eletrônica dos Arquivos Brasileiros de Oftalmologia com textos completos está disponível em:}

- $\mathbf{A B O}$ - Arquivos Brasileiros de Oftalmologia http://www.abonet.com.br

- SciELO - Scientific Electronic Library Online http://www.scielo.org

- Free Medical Journals - http://www.freemedicaljournals.com 